\title{
The Use of Road Microsimulation Software within BIM Environments: A Preliminary Assessment
}

\author{
Marilisa Botte (D), Amedeo Zampi iD, Cristina Oreto $\left(D\right.$, and Luca D’Acierno ${ }^{1}$ \\ Department of Civil, Architectural and Environmental Engineering, Federico II University of Naples, Via Claudio 21, \\ Naples 80125, Italy \\ Correspondence should be addressed to Luca D’Acierno; luca.dacierno@unina.it
}

Received 31 July 2020; Accepted 31 July 2021; Published 25 August 2021

Academic Editor: Gonçalo Homem de Almeida Correia

Copyright ( $\odot 2021$ Marilisa Botte et al. This is an open access article distributed under the Creative Commons Attribution License, which permits unrestricted use, distribution, and reproduction in any medium, provided the original work is properly cited.

The use of Building Information Modelling (BIM) is becoming increasingly adopted worldwide to support the creation and management of digital environments in which physical and functional features of a facility are modelled. Due to its characteristic flexibility and interoperability, it rapidly extends beyond the boundaries of applications for which it was originally conceived, thus being implemented in other contexts such as the infrastructure sector. Indeed, during each phase of the construction process for building a linear infrastructure, a suitable design of a traffic control plan to optimising traffic flows proves crucial, both for drivers and builders. In this context, there arises the need to perform a comprehensive analysis, combining infrastructure design and building issues with transportation theory principles. For this purpose, the paper proposes a comparative analysis of two different approaches relying on BIM-based tools, namely integrated (i.e., BIM environments with an in-built module for transport simulation tasks) and an interfaceable solution (i.e., BIM environments offering a customised interface for external mobility simulation software). Specifically, after an overview of the issues involved, the two solutions analysed are customised according to the software packages adopted, and a numerical application is carried out. The goal of the paper is to perform a preliminary analysis on the potential of such frameworks by means of comparative evaluation aimed at identifying pros and cons of the two approaches, as well as the best field of application, according to the effort required from the designer, the degree of flexibility offered, and the target pursued.

\section{Introduction}

With the advent of Information and Communication Technologies (ICTs), cities have become increasingly smart and interconnected. This leads to several complex design and management issues being addressed which require suitable approaches for implementation within the field of Architecture, Engineering, and Construction (AEC). In this context, one of the most promising and widely applied methodologies is Building Information Modelling (BIM) [1], which may be defined as a procedure of planning, building, and handling a facility relying on computerised object-orientated data. Given the potential of such a tool, BIM has been extended to applications for which it was not formerly conceived (e.g., the infrastructure sector $[2,3])$. Indeed, in transportation contexts, the implementation of BIM may concern the design and construction of airports [4-6], railways [7-9], and embankments [10]. Consequently, the construction industry within the European Union has progressively used this methodology [11], and elsewhere [12-14].

The success of such a design and management technology lies in its multidisciplinary and interoperability features. Indeed, BIM can be integrated with other engineering tools and allows the temporal dimension to be embedded in the analysis. The latter assumes a key role in supporting traffic control planning during construction works. Indeed, an optimisation process of traffic flows is required during construction works, traffic conditions needing to be managed to avoid inconvenience for drivers and traffic safety. In this context, the necessity of conceiving and handling a proper interface between BIM-based software and transportation theory occurs. 
There are several BIM-based tools used in the construction sector, developed by the main software houses, such as Autodesk and Bentley Systems. The choice of the most suitable tool depends on the purpose of use, that is, clash analysis, structural analysis, energy analysis, cost estimation, and so on $[15,16]$. Some BIM-based environments integrate a mobility simulation module, while others allow for an interface with a specific transport simulation software package.

In the latter case, specific procedures for import/export need to be set and compatible file formats are shared. However, it appears evident that exploiting such interoperability features to the full is no mean feat. Indeed, interoperability does not mean a simple interchange of information, such as geometric characteristics but also the interchange of rationale [17]. This implies the need for an indepth understanding of the logic and models implemented in the software packages adopted. Within this context, the proposed research study analyses different approaches for dealing with the issue of managing traffic control planning during construction works. In particular, methodologies which diversely adopt BIM technologies are considered, thus investigating the potential behind such a promising tool. More specifically, an integrated (i.e., BIM environments with an in-built module for transport simulation tasks) and an interfaceable solution (i.e., BIM environments offering a customised interface for external mobility simulation software) are tested and a comparative evaluation is provided.

The rest of the paper is organised as such: Section 2 presents an overview of transportation systems simulation; Section 3 illustrates different approaches on which a design engineer can rely for dealing with traffic control planning during construction works on a linear infrastructure and related issues; Section 4 tests such alternative methods for the purpose of comparison; and finally, Section 5 draws conclusion and outlines research prospects.

\section{Simulation of Mobility Systems}

Transport systems are conceived to generate travelling options thus meeting travel demand requirements. Therefore, the key aim of transport simulation software is to model the interactions between travel demand and supply facilities, both in design and management phases. The goal is to provide the temporal and spatial distribution of traffic flows on the network, thus supporting any kind of decisional process involving transport systems. Specifically, as shown by Cascetta [18], the deployment of a transportation system includes the following steps: (i) identification of the study area; (ii) discretisation of the study area into traffic zones; (iii) determination of significant infrastructures and services; (iv) definition of the supply model; (v) definition of the demand model; and (vi) modelling of the interaction between supply and demand characteristics.

Beside preparation phases, supply facilities are described by means of two models, namely topological and analytical. The topological one relies on the graph theory [19-21], while the analytical structure is based on the following models: flow propagation model (identifying the connection between link and path flows), link performance model, based on cost functions (describing how the flow affects the performance of a link), and path performance model (identifying the relationship between link and path performance).

The transportation demand model considers four different choice dimensions: whether or not to make the journey (i.e., trip generation model), the destination to be reached (i.e., trip distribution model), by which mode (i.e., mode choice model) and through which path (i.e., route choice model). Travel demand needs to be characterised both in spatial and temporal terms. In particular, spatial representation is based on a matrix structure, that is, the origin-destination matrix. With regard to the temporal dimension, instead, intra-period stationarity or intra-period dynamics assumptions can be made.

Finally, interactions between supply and demand features are simulated by means of the so-called assignment models which provide system performance thus allowing any kinds of evaluation. These models can be deterministic or stochastic, as well as can consider the phenomenon of congestion (i.e., the dependence of link performance on link flows) or not. Moreover, also the dependence of OD flows on path costs may be modelled. However, further details about assignment models can be found in Cascetta [18]; what follows, instead, is focused on traffic flow simulation models.

Given the goal of the proposed approach (i.e., managing traffic control planning during construction works), nonstationary models are considered. According to the level of detail implemented, such models can be classified into macroscopic, mesoscopic, and microscopic. In macroscopic models, traffic streams are represented as a partially compressible fluid, composed of infinitesimal parcels; indeed, this approach is called fluid approximation. Mesoscopic models, instead, represent traffic as groups of vehicles. Finally, in microscopic models, the trip of every single vehicle is reproduced by means of models which explicitly simulate the speed-adjusting behaviour (e.g., gap-acceptance, car following, overtaking, lane changing).

The models described above are generally implemented by means of simulation software packages. For our purpose, a microscopic simulation is required and, amongst others, suitable commercial software packages are Aimsun [22], Sumo [23], and PTV Vissim [24].

It is worth noting that the main issue to address in adopting microsimulation software is the calibration phase according to the context analysed. This tuning step is fundamental to obtain reliable and accurate results.

\section{Alternative Options Made Available for Design Engineers}

As stated above, the purpose is to investigate the potential offered by BIM-based tools for supporting the management of traffic flow during construction works. Indeed, changes to transport infrastructure required during construction works alter ordinary conditions of traffic flow and this transition phase needs to be properly addressed to avoid inconvenience to drivers. For example, when a two-way road has to be maintained or is affected by the construction of an overhead bridge, 
in order to avoid a complete road closure, various construction steps have to be implemented and, for each of them, a specific strategy in the management of traffic flows is required.

Therefore, it appears fundamental to integrate BIM tools with transportation simulation approaches. In this section, three alternative options available for design engineers are presented to compare them and analyse the strengths and weaknesses of each approach.

BIM-based tools offer different options for mobility simulation (see Figure 1). In particular, besides the standard solution in which the designer has to deal with the integration of the two methods (i.e., BIM and mobility simulation), the following conditions appear more noteworthy:

(i) BIM-based tools with an in-built module for transport simulation tasks (i.e., integrated approach)

(ii) BIM-based tools offering a customised interface for external mobility simulation software packages (i.e., interfaceable approach)

Clearly, for each solution, different software packages can be adopted. However, commercial software is often used as a black-box because in-built adopted procedures are generally unknown to the designers. Instruction manuals can give information on the models adopted but without specifying numerical values of the parameters involved or, even if indicated, cannot be changed. The tool in question could be unhelpful in the analysis of specific contexts. In light of the above, it appears fundamental to investigate the logic and models implemented in the adopted tools, thus being able to fully control them and evaluate the possibility of customising them to the specific context analysed. Obviously, in the case of tools or software packages accompanied by documentation, explicitly explaining models and parameters implemented, the proposed analysis would be trivial.

The proposal to compare the aforementioned solutions and define which offers a more reliable and accurate analysis of traffic flows consists of a simulation-based procedure. It is worth noting that other methodologies such as artificial neural networks would be unsuitable for this purpose because they would provide a black-box tool as well, without offering the possibility of selecting the functional forms and the values of related parameters according to the target.

The proposed methodology consists of performing several simulations with given input parameters, thus obtaining related outputs and deriving the mathematical relationship linking them (i.e., inputs and outputs). It is thus possible to find the relationships adopted among significant traffic flow variables, as well as which cost functions are implemented in the model. Furthermore, the route choice model embedded in the procedure can thereby be identified.

In order to find the function which best fits some sampling data, a three-step procedure is generally followed, whose phases are as follows: specification (i.e., identifying functional form), calibration (i.e., estimating numerical values of parameters), and validation (i.e., performing statistical tests to evaluate the goodness of fit). However, in our case, data to be interpolated are provided through a simulation procedure and the crucial step is represented by the calibration phase. Functional forms are known from transport theory because they are related to physical phenomena (such as vehicle motion) and/or behavioural aspects (such as interactions between vehicles and/or users). The fact that functional forms, as well as numbers and features of required parameters, are given by the literature means that, once the parameters involved have been calibrated, to validate the derived relationship, only global statistical tests are performed, neglecting statistics on parameters.

The model has to work in order to comply with the Macroscopic Fundamental Diagram (MFD), expressing the relationship between any pair of the following variables: flow, density, and speed. It is reproduced, qualitatively, in Figure 2.

According to the MFD, flow can be equal to zero under two assumptions: no vehicles are on the road (i.e., density is null) or when vehicles are in a stop condition (i.e., speed is null). In the first case, the speed assumes the theoretical maximum, the so-called free-flow speed, $v_{0}$, while in the second case, the density assumes the theoretical maximum, i.e., the so-called jam density, $k_{\text {jam }}$. The peak of the speedflow (and density-flow) curve represents road capacity, i.e., the theoretical maximum flow, Cap. The corresponding speed and density are referred to as the critical speed, $v_{c}$, and critical density, $k_{c}$. Therefore, besides the capacity, any flow value can occur under two different conditions: low speed and high density (i.e., unstable state) and high speed and low density (i.e., stable state). There are several mathematical models expressing the relationships among these variables, such as that proposed by Greenshields [25], from which travel time on a running link can be derived.

In alternative, travel time on a link can be computed as a function of flow and free-flow speed. The most widely used function expressing such a relation, generally associated with the stable regime, is the BPR cost function, proposed by U.S. Bureau of Public Roads in 1964 [26].

Finally, the implemented route choice model needs to be investigated. Generally, during construction road works, this is the most greatly affected travel demand choice dimension. Route choice models are based on random utility theory [27]. Amongst others, the most widely used models are the Multinomial Logit model [28], or the Probit model [29]. The former has the remarkable benefit of relying on a closed form; however, in some contexts, it could provide a biased outcome due to the property of independence from irrelevant alternatives. This has led to many variants of the model, aimed at overcoming the drawback by preserving the closed form as, for instance, in Nested Logit [30, 31], C-Logit [32], and CoRUM [33]. The Probit model, instead, for deriving route choice probabilities and related path flows, relies on sampling techniques of path random residuals based on the Monte Carlo method [34-36].

\section{Customisation of the Proposed Methodology to Existing Tools and Its Application to Toy Networks}

In this section, the proposed methodology is customised to the specific existing software packages, thus testing the suggested approach and showing its feasibility. As shown by 


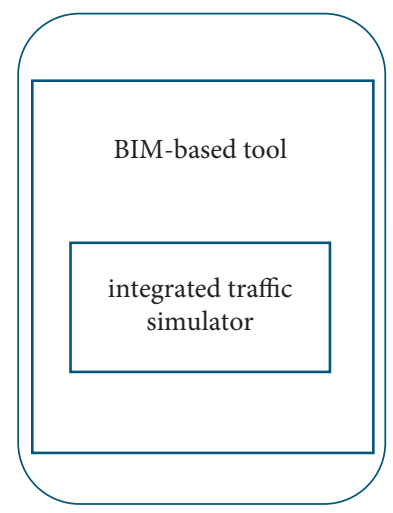

(a)

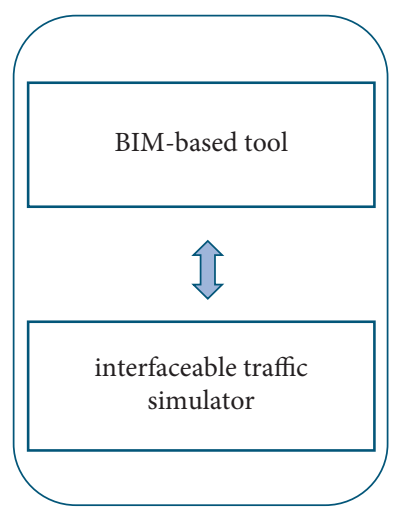

(b)

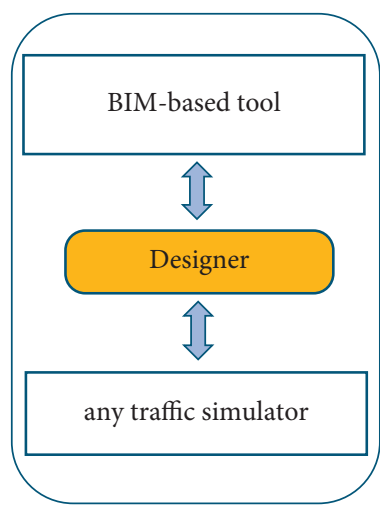

(c)

FIgURE 1: Alternative options for designer engineers: (a) integrated solution; (b) interfaceable solution; and (c) standard solution.

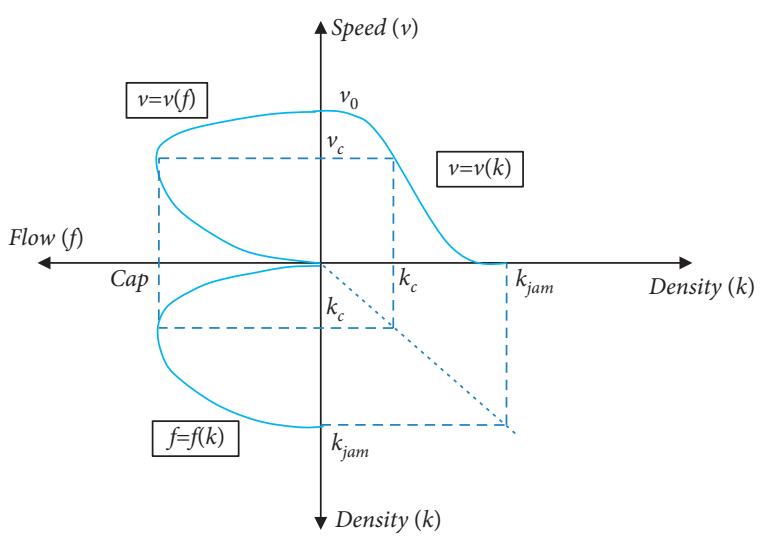

Figure 2: Fundamental diagram of traffic flow

Figure 3, among the commercial BIM-based software, we analyse Autodesk InfraWorks 360 and Open Roads Concept Station by Bentley. The former embeds an in-built mobility simulation module, thus offering an integrated solution which does not need any additional transportation software. The latter, instead, can be adopted jointly with the microsimulation software PTV Vissim thanks to the presence of a suitable interface window suitably set up by the producer.

Therefore, leaving aside the standard solution, in which it is the designer who acts as the interface between the two environments (i.e., BIM and traffic simulation), the other two approaches can be customised according to Figure 3. At this point, the proposed simulation-based methodology is applied in the case of approach 1 (i.e., integrated solution) and approach 2 (i.e., interfaceable solution), thus comparing them and deriving the pros and cons of each method.

By linking input data (given a priori) with simulation outputs using a calibration procedure, the following issues are investigated: the relation between flow and speed; the implemented cost function, expressing the relationship between travel time and flow; the probability values provided by the adopted route choice model.

To define the relations linking flow and speed and the cost functions implemented in the models, a network made up of a single-link connecting an OD pair is considered (Figure 4(a)). By contrast, in order to investigate the route choice model embedded in the simulation software packages involved in the two approaches, a network with two links with different features, connecting a single OD pair, is analysed (Figure 4(b)). The choice of adopting such networks is made for the sake of simplicity: with such frameworks, link flows and path flows are equivalent.

In order to investigate relations between flow and speed and cost functions, the network shown in Figure 4(a) is characterised according to three network configurations, differing in link length, number of lanes, lane width, and free-flow speed (see Table 1).

Therefore, after fixing the geometrical and functional features of the infrastructure, several simulations are run by increasing the OD flow involved.

As a preliminary step, the travel time in free-flow conditions, indicated as $t_{0}$, can be derived as the ratio between link length and free-flow travel speed. Then, for each simulation, having fixed a specific road section $s$, the number of vehicles (indicated as $N$ ) which passes through $s$ during the simulation time interval $\Delta t$ can be obtained. By means of $N$, the related flow, $f$, can be computed as follows:

$$
f=\frac{N}{\Delta t-t_{0}} \text {. }
$$

A further output of the simulation procedure is the average travel time, from which, given the link length, the average travel speed, indicated as $v$, can be derived.

For each network configuration, 15 simulations are performed, obtaining 15 "link flow-speed" pairs on the basis of which related functions $f=f(v)$ are estimated. As already pointed out, the specification of this function is known from traffic flow theory. It is a parabola passing through the origin with a horizontal axis and the concave section towards the origin, which can be expressed by the following equation:

$$
f=a v^{2}+b v+c
$$

with $a<0$ and $c=0$.

Given the functional form, the calibration and validation procedures are carried out. For the calibration step, function parameters (i.e., $a$ and $b$ ) are estimated in order to fit simulation data. 


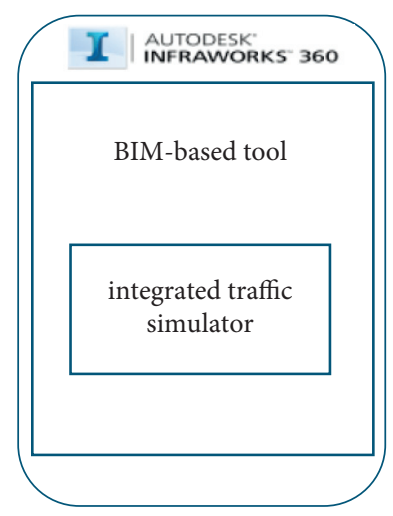

(a)

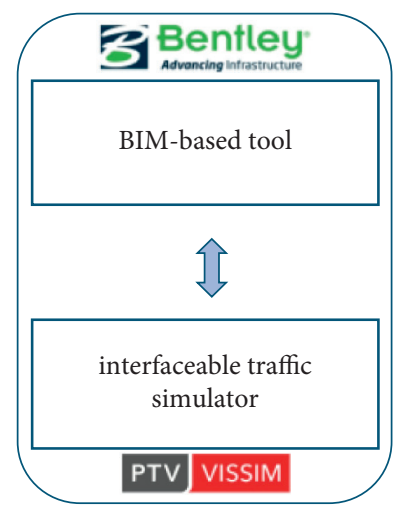

(b)

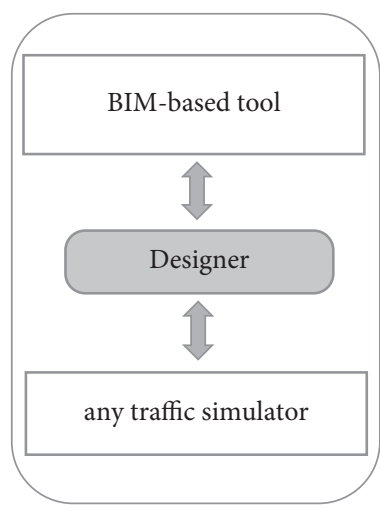

(c)

FIGURE 3: Alternative options for designer engineers: (a) customised integrated solution; (b) customised interfaceable solution; and (c) standard solution.

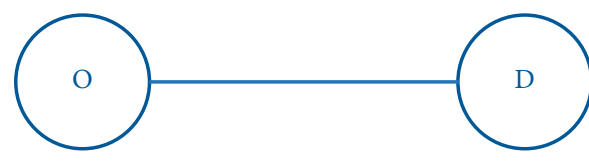

(a)

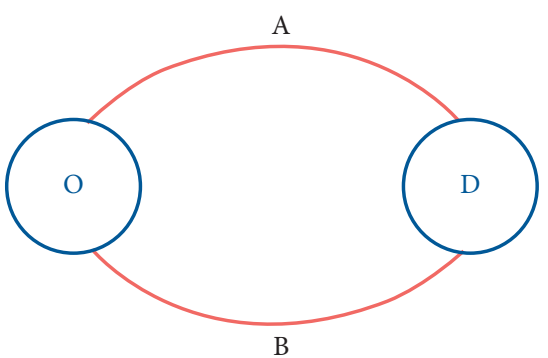

(b)

FIGURE 4: Test networks: (a) single-link framework and (b) double-link framework.

TABLE 1: Network configurations.

\begin{tabular}{lcccc}
\hline Configuration & Link length $(\mathrm{m})$ & Number of lanes & Lane width $(\mathrm{m})$ & Free-flow speed $(\mathrm{km} / \mathrm{h})$ \\
\hline 1 & 5180 & 1 & 3.50 & 80 \\
2 & 4200 & 1 & 3.50 & 40 \\
3 & 3570 & 2 & 3.50 & 40 \\
\hline
\end{tabular}

Numerical results for the three network configurations analysed and the two approaches analysed are shown in Table 2 and Figures 5 to 10. In particular, Figures 5 and 6 compare the integrated and interfaceable solutions concerning network configuration 1; Figures 7 and 8 concerning network configuration 2; Figures 9 and 10 concerning network configuration 3 .

Instead, for the validation phase, some global statistical tests are performed, namely coefficient of determination $\mathfrak{R}^{2}$, adjusted $\mathfrak{R}^{2}$ (indicated as $\overline{\mathfrak{R}}^{2}$ ), and F-test (indicated as F), as reported in Tables 3 to 5 for each network configuration.

The further issue to be investigated concerns the link performance model which simulates the dependence of link performance on related flows. As can be seen from the above, we are in the stable regime and this, according to traffic flow theory, allows us to assume the BPR as the adopted cost function. Travel time on link $l$, indicated as $t_{l}$, may be expressed as depending on the related link flow, indicated as $f_{l}$, that is,

$$
t_{l}=t_{l 0} \cdot\left[1+\alpha \cdot\left(\frac{f_{l}}{\operatorname{Cap}_{l}}\right)^{\beta}\right]
$$

where $t_{l o}$ represents free-flow travel time on link $l$; Cap $l$ represents the capacity of link $l ; \alpha$ and $\beta$ are parameters to be calibrated.

In the case of a microsimulation approach, capacity is estimated with a lane-based method, that is, as a function of the number of lanes characterising the link, $n_{l}$, according to a parameter $\gamma$ which generally needs to be calibrated as well.

Therefore, it can be stated that,

$$
\mathrm{Cap}_{l}=\gamma \cdot n_{l} \text {. }
$$

In our case, since only one link makes up the network analysed, equation (3) can be rewritten as follows:

$$
\frac{t}{t_{0}}=1+\alpha \cdot\left(\frac{f}{\text { Cap }}\right)^{\beta} \text {. }
$$

As in the procedure followed above, for the network configurations analysed, several simulations are run by 
TABLe 2: Numerical values of calibrated parameters of function $f=f(v)$.

\begin{tabular}{lcccccc}
\hline & \multicolumn{2}{c}{ Network configuration 1 } & \multicolumn{2}{c}{ Network configuration 2 } & \multicolumn{2}{c}{ Network configuration 3 } \\
& $a$ & $b$ & $a$ & $b$ & $b$ & $a$ \\
\hline Integrated solution & -1.090 & 92.090 & -5.953 & 225.917 & -11.556 & 464.701 \\
Interfaceable solution & -1.239 & 110.492 & -12.819 & 525.330 & -29.857 & 1218.450 \\
\hline
\end{tabular}

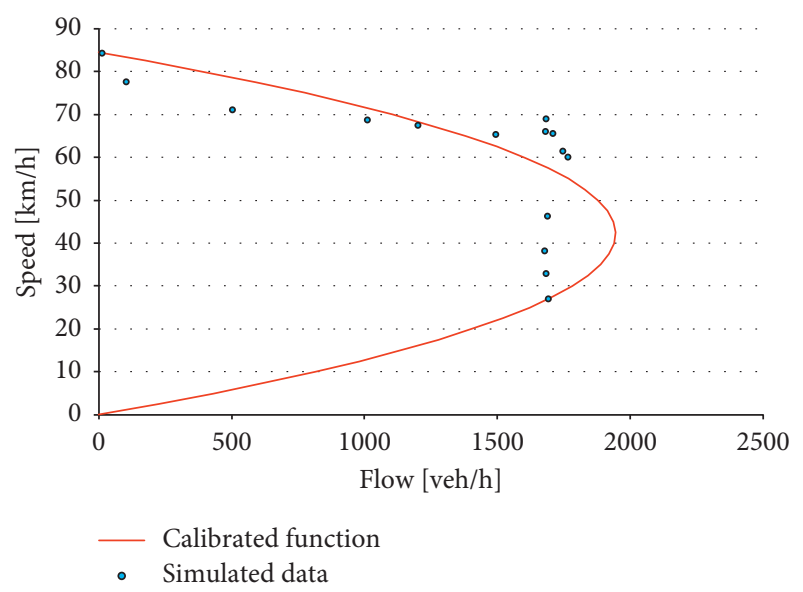

Figure 5: Calibrated function $f=f(v)$ in the case of network configuration 1 for the integrated solution.

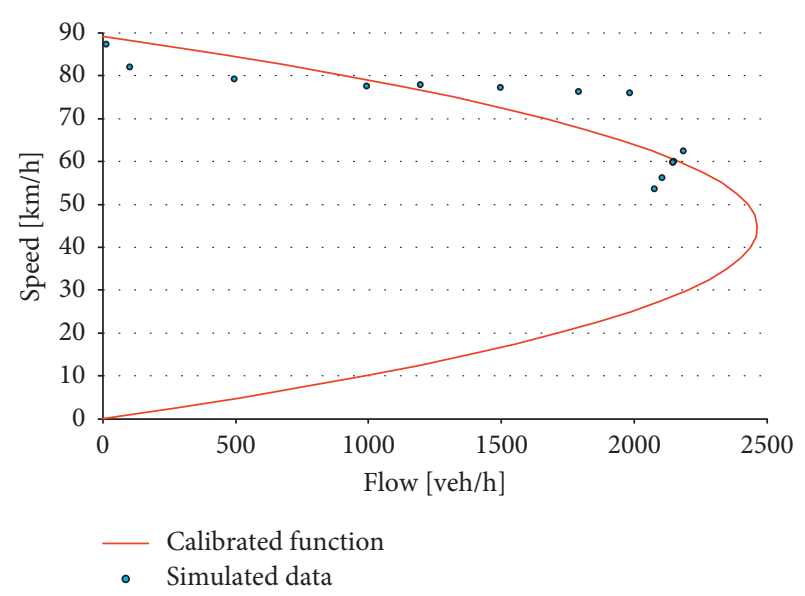

Figure 6: Calibrated function $f=f(v)$ in the case of network configuration 1 for the interfaceable solution.

increasing the OD flows involved. In this way, a total of 45 $\left(f / \mathrm{Cap} ; t / t_{0}\right)$ pairs are obtained and interpolated by calibrating function parameters (i.e., $\alpha, \beta$, and $\gamma$ ) in order to fit the simulation data.

It is worth noting that, in our analysis, parameter $\gamma$ is calibrated only in the case of the integrated solution. By contrast, in the case of the interfaceable approach, the default value implemented in the traffic simulator adopted (i.e., PTV Vissim) is considered, that is $\gamma=900$.

Therefore, the numerical values of the calibrated parameters are, in the case of the integrated solution, $\alpha=0.1661, \beta=3.6483$, and $\gamma=1040$ while, in the case of the interfaceable solution, $\alpha=0.0002$ and $\beta=8.4411$ are obtained. Calibrated BPR functions are shown in Figures 11

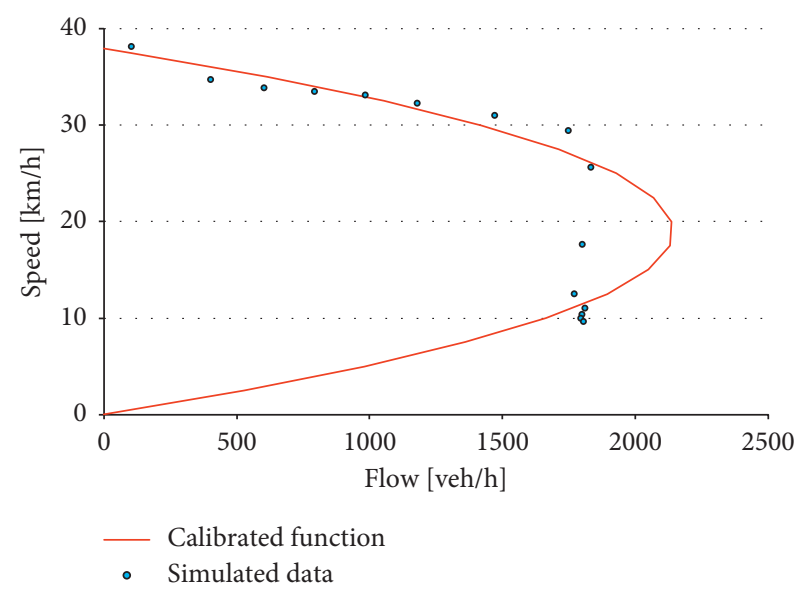

FIgure 7: Calibrated function $f=f(v)$ in the case of network configuration 2 for the integrated solution.

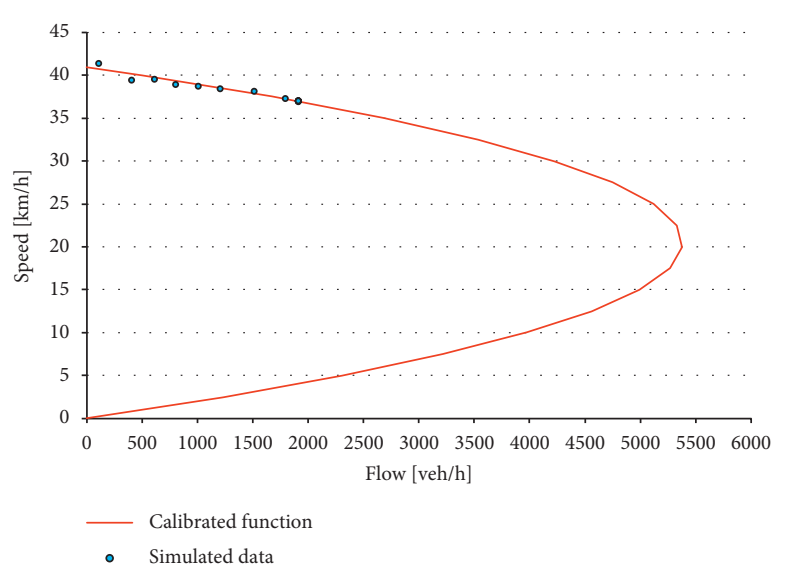

FIgURE 8: Calibrated function $f=f(v)$ in the case of network configuration 2 for the interfaceable solution.

and 12 , respectively, for the integrated and interfaceable solution.

The aforementioned global statistical tests are also performed as a validation step. The related results are shown in Table 6 for the two approaches analysed.

Finally, the route choice models implemented in the software packages involved in the two presented approaches (i.e., integrated and interfaceable) are investigated.

According to random utility theory [27], the functional formulation of a multinomial logit model is assumed. It considers that the random residuals are independently and identically distributed according to a Gumbel random variable with zero mean and parameter $\theta$. However, given the adopted network (see Figure 4(b)), a binomial Logit 


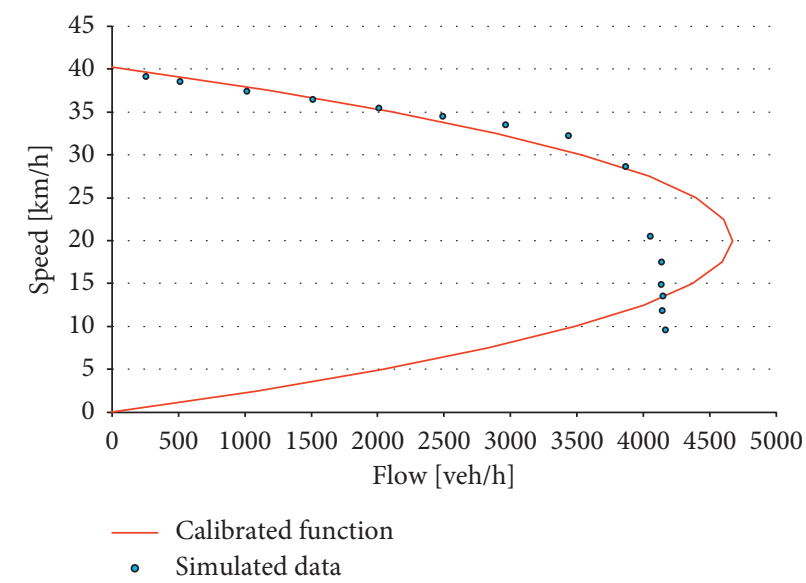

FIgURE 9: Calibrated function $f=f(v)$ in the case of network configuration 3 for the integrated solution.

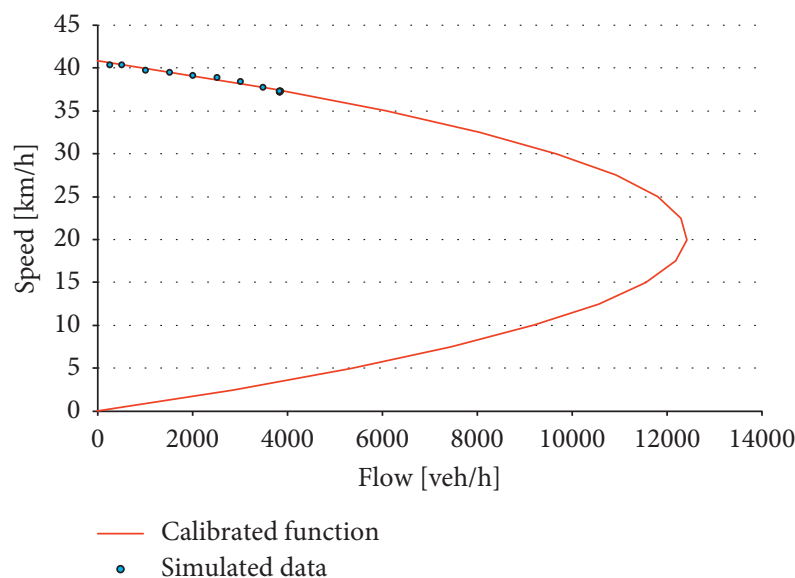

Figure 10: Calibrated function $f=f(v)$ in the case of network configuration 3 for the interfaceable solution.

TABLE 3: Global statistical tests related to function $f=f(v)$ in the case of network configuration 1 .

\begin{tabular}{lccccc}
\hline & $\mathfrak{R}^{2}$ & $\mathfrak{R}^{2}$ & \multicolumn{2}{c}{$\begin{array}{c}\text { F-test } \\
\text { Confidence level (\%) }\end{array}$} \\
\hline Integrated solution & & & $F$ value & Threshold & 99.90 \\
Interfaceable solution & 0.694 & 0.644 & 36.922 & 21.137 & 96.00 \\
\hline
\end{tabular}

TABLE 4: Global statistical tests related to function $f=f(v)$ in the case of network configuration 2.

\begin{tabular}{lccccc}
\hline & $\mathfrak{R}^{2}$ & $\mathfrak{R}^{2}$ & & \multicolumn{2}{c}{$\begin{array}{c}\text {-test } \\
\text { Confidence level (\%) }\end{array}$} \\
\hline Integrated solution & & & $F$ value & Threshold & 99.90 \\
Interfaceable solution & 0.958 & 0.951 & 141.557 & 21.137 & 95.95 \\
\hline
\end{tabular}

TABLE 5: Global statistical tests related to function $f=f(v)$ in the case of network configuration 3 .

\begin{tabular}{lccccc}
\hline & $\mathfrak{R}^{2}$ & $\mathfrak{R}^{2}$ & & \multicolumn{2}{c}{$\begin{array}{c}\text {-test } \\
\text { Confidence level (\%) }\end{array}$} \\
\hline Integrated solution & 0.975 & 0.970 & 202.672 & 21.137 & 99.90 \\
Interfaceable solution & 0.889 & 0.857 & 349.45 & 31.555 & 99.90 \\
\hline
\end{tabular}




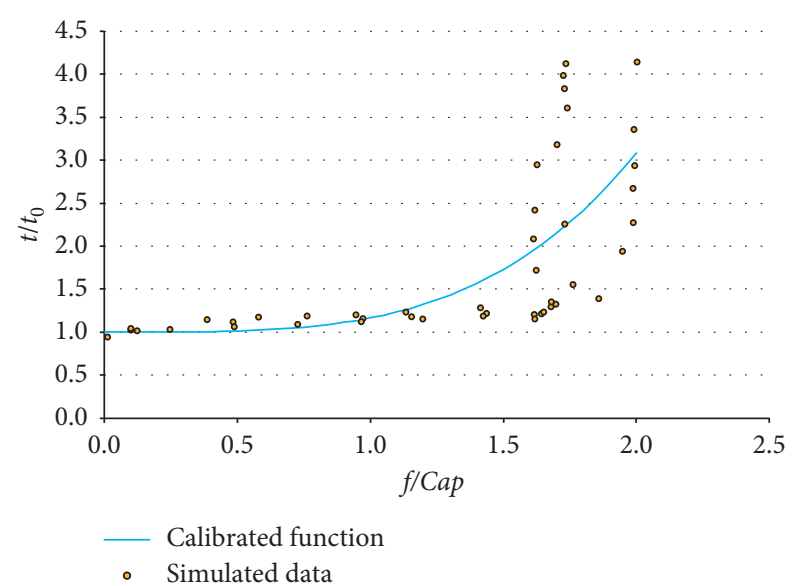

FIgURE 11: Calibrated BPR function for the integrated solution.

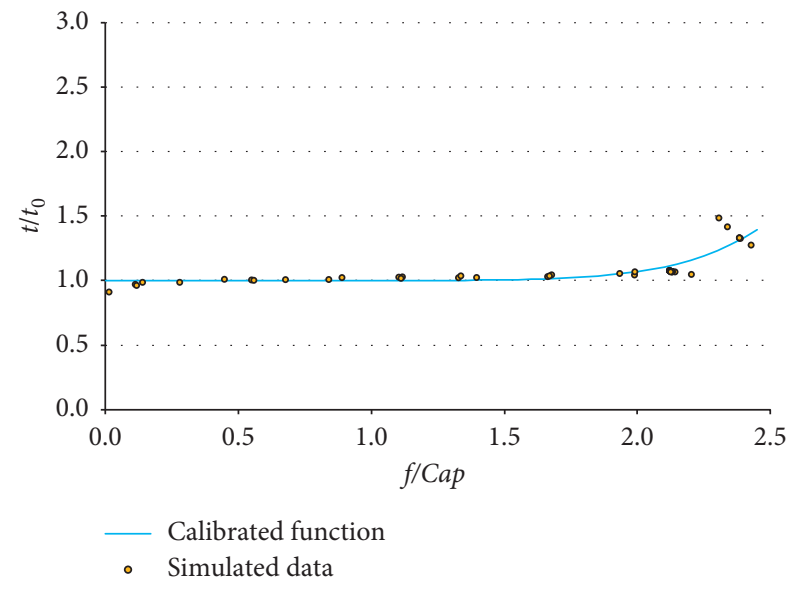

FIGURE 12: Calibrated BPR function for the interfaceable solution.

structure is considered. As shown by Cascetta [18], it can be expressed as follows:

$$
\begin{aligned}
p(A) & =\frac{\exp \left(V_{A} / \theta\right)}{\exp \left(V_{A} / \theta\right)+\exp \left(V_{B} / \theta\right)}, \\
& =\frac{1}{1+\exp \left[\left(V_{B}-V_{A}\right) / \theta\right]}, \\
p(B) & =\frac{\exp \left(V_{B} / \theta\right)}{\exp \left(V_{A} / \theta\right)+\exp \left(V_{B} / \theta\right)}, \\
& =\frac{1}{1+\exp \left[\left(V_{A}-V_{B}\right) / \theta\right]},
\end{aligned}
$$

where $p(A)$ is the route choice probability related to path $A$; $p(B)$ is the route choice probability related to path $B ; V_{A}$ is the systematic utility related to path $A ; V_{B}$ is the systematic utility related to path $B$; and $\theta$ is the aforementioned parameter of the Gumbel distribution.

Systematic utilities represent a measure of user convenience in adopting a certain alternative and are generally expressed as a linear combination of attributes and weights. More details on this can be found in Cascetta [18]; however, in the following, we consider systematic utilities as a function of travel times according to a parameter $\lambda$ which expresses the importance associated to travel time by users, i.e.,

$$
\begin{aligned}
& V_{A}=-\lambda \cdot t_{A}, \\
& V_{B}=-\lambda \cdot t_{B},
\end{aligned}
$$

where $t_{A}$ is the travel time associated to path $A$ and $t_{B}$ is the travel time associated to path $B$. The minus sign is used because travel time represents an inconvenience for users: the greater the travel time, the lower the utility that users associate to that alternative. Therefore, also in this case, several simulations are run by changing network features (i.e., length and free-flow speed of link $A$ and link $B$ ) and OD flows involved, amounting to a total of 30 records.

Simulation outputs of concern are as follows:

$N_{A}$ : number of vehicles passing through section $s_{A}$ on link $A$ during the simulation time interval $\Delta t$, according to which the related flow $f_{A}$ can be obtained by means of equation (1);

$N_{B}$ : number of vehicles passing through section $s_{B}$ on link $B$ during the simulation time interval $\Delta t$, according to which the related flow $f_{B}$ can be obtained by means of equation (1).

Therefore, simulated choice probability values may be computed as

$$
\begin{aligned}
& p(A)=\frac{N_{A}}{N_{A}+N_{B}}, \\
& p(B)=\frac{N_{B}}{N_{A}+N_{B}} .
\end{aligned}
$$

In the following, for the sake of brevity, we focus on the results related to $p(A)$. However, this does not affect the outcome in any way, since choice probabilities $p(A)$ and $p$ $(B)$ are dependent values (i.e., $p(A)+p(A)=1$ ).

According to equation (6), the binomial logit model can be rewritten as follows for the probability of choosing path $A$ :

$$
p(A)=\frac{1}{1+\exp \left[-\lambda\left(t_{B}-t_{A}\right) / \theta\right]},
$$

with ratio $\lambda / \theta$ to be calibrated.

Since, in the adopted network, there is a coincidence between links and paths, $t_{A}$ also represents the travel time associated to link $A$, just as $t_{B}$ represents the travel time associated to link $B$. This implies that they can be computed according to the BPR function defined above. Therefore, also in this case, the model parameter (i.e., ratio $\lambda / \theta$ ) is calibrated and statistical tests are performed for the validation phase. In the case of the integrated solution, $\lambda / \theta$ is equal to 0.70 while, in the case of the interfaceable solution, $\lambda / \theta=1.32$. Table 7 shows global statistical tests for the approaches analysed.

Moreover, in order to perform a transportation science analysis, it appears interesting to provide a comparison between simulation and model data as a function of the difference between systematic utilities of the two alternative paths. Figures 13 and 14 report this 
TABLE 6: Global statistical tests in the case of the BPR cost function.

\begin{tabular}{lccccc}
\hline & $\mathfrak{R}^{2}$ & $\mathfrak{R}^{2}$ & & \multicolumn{2}{c}{-test } \\
& & & $F$ value & Threshold & Confidence level (\%) \\
\hline Integrated solution & 0.473 & 0.435 & 18.579 & 9.159 & 99.90 \\
Interfaceable solution & 0.694 & 0.644 & 36.922 & 21.137 & 99.90 \\
\hline
\end{tabular}

TABLE 7: Global statistical tests in the case of the route choice model.

\begin{tabular}{lccccc}
\hline & $\mathfrak{R}^{2}$ & $\overline{\mathfrak{R}}^{2}$ & \multicolumn{2}{c}{-test } \\
& & & $F$ value & Threshold & Confidence level (\%) \\
\hline Integrated solution & 0.784 & 0.768 & 367.761 & 15.485 & 99.90 \\
Interfaceable solution & 0.993 & 0.992 & 763.422 & 15.485 & 99.90 \\
\hline
\end{tabular}

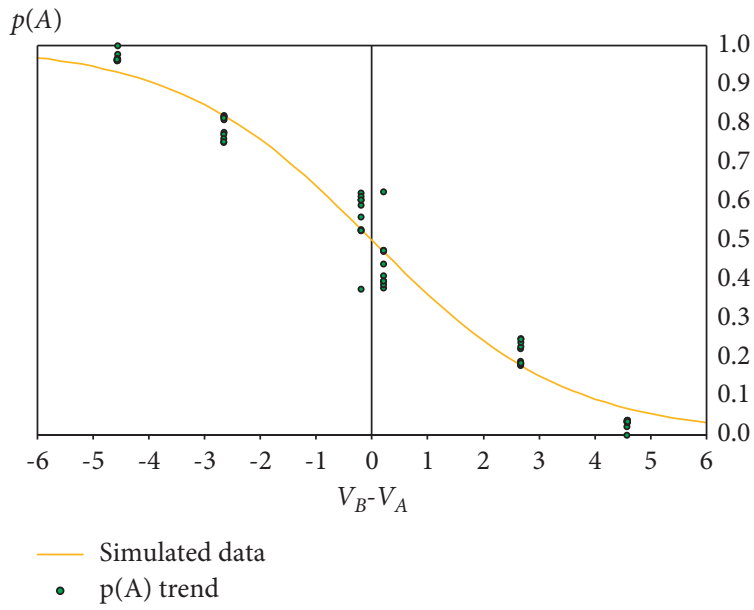

FIgURE 13: Trend of choice probability $p(A)$ as a function of $\left(V_{B}-V_{A}\right)$ for the integrated solution.

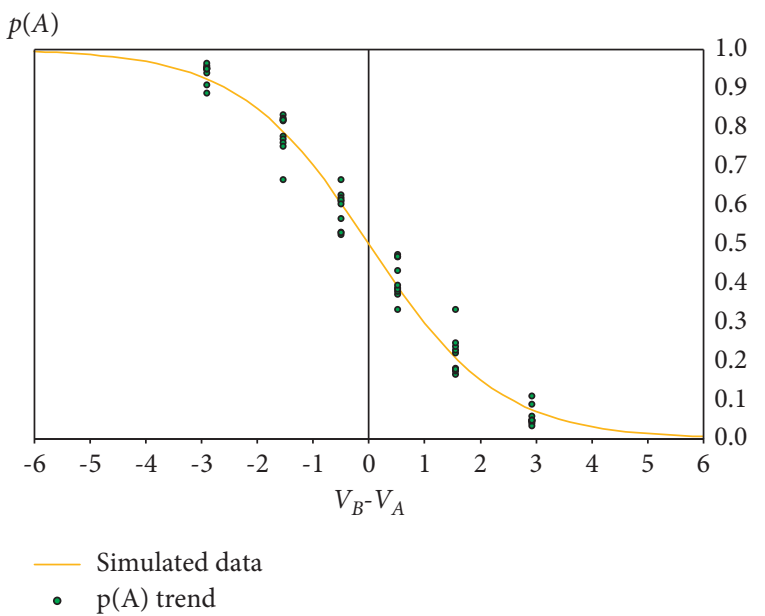

FIgURE 14: Trend of choice probability $p(A)$ as a function of $\left(V_{B}-V_{A}\right)$ for the interfaceable solution.

comparative view for a range of the difference between $V_{B}$ and $V_{A}$ equal to $[-6 ; 6]$, respectively, for the integrated and interfaceable solutions. Finally, Figures 15 and 16 compare the outcome of the models embedded in the design approaches analysed (i.e., integrated and interfaceable), respectively, in terms of BPR function cost and route choice model.
As can be seen, regarding the integrated solution, both the BPR function and route choice model appear feasible; parameters of BPR are very close to the default values (i.e., $\alpha=0.15$ and $\beta=4)$. By contrast, regarding the interfaceable solution in which the mobility simulation is performed by PTV Vissim, the BPR presents an unusual trend while the route choice model tends to a deterministic condition. 


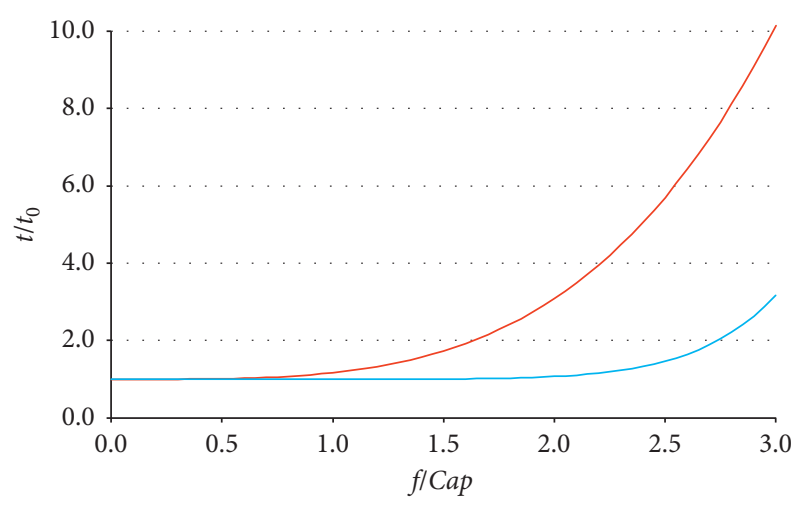

- BPR function in the case of the integrated solution _ BPR function in the case of the interfaceable solution

FIGURE 15: Comparison between the two approaches analysed (i.e., integrated vs interfaceable solution) in terms of BPR function cost.

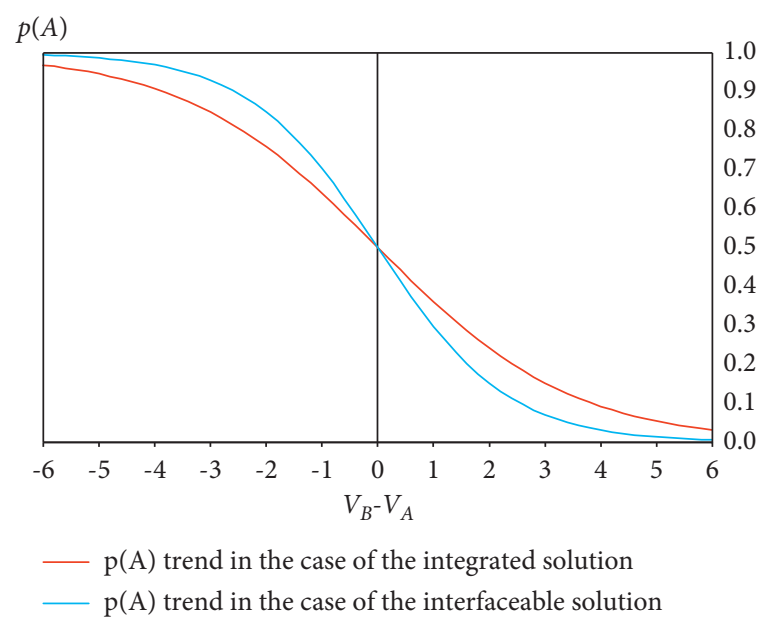

FIGURE 16: Comparison between the two approaches analysed (i.e., integrated vs interfaceable solution) in terms of route choice model.

However, it is worth noting that the issue of a biased trend of the cost function is due to the lack of a proper preliminary tuning phase for the traffic simulator, rather than to the ineffectiveness of the tool adopted.

\section{Conclusion and Research Prospects}

The proposed work analyses the potential of BIM-based tools in managing traffic flows during construction works on a linear infrastructure. As modifications to transport supply affect traffic flows, it is fundamental to consider a customised traffic plan during such transition phases.

In particular, an integrated solution (i.e., BIM-based tools with an in-built module for transport simulation tasks) and an interfaceable approach (i.e., BIM-based tools offering a customised interface for external mobility simulation software) are analysed. A comparison is carried out based on the following elements embedded in the model: relationships among traffic fundamental variables, cost functions, and route choice models. Autodesk InfraWorks 360 is considered for testing the integrated option, while the combination of Open Road Concept Station and PTV Vissim microsimulation software is used for the interfaceable solution.

On comparing simulation results, several considerations arise. First, the numerical application confirms the importance of a suitable preliminary calibration phase for obtaining reliable solutions from traffic microsimulation software. Therefore, the interfaceable solution appears viable only if the designer has the possibility (in terms of time and, therefore, budget) to pay due attention to this crucial step; otherwise, the outcome proves biased. Moreover, the interfaceable solution presents a higher degree of flexibility because the designer may choose, according to requirements, among different software packages, as long as they are compatible. Furthermore, it is worth comparing the two approaches on the basis of the target pursued. Indeed, in the case of a preliminary design phase, where a feasibility assessment is needed, the adoption of integrated solutions appears a good compromise between efforts required and desired accuracy for the results obtained. On the contrary, if the designer has to deal with a thorough design and building step, the interfaceable solution appears the most suitable option since, against the initial calibration procedure required for a traffic simulator and a small intermediate step for dealing with the interface, it allows a more accurate evaluation to be performed.

With regard to research prospects, we suggest performing additional investigations to test different BIM-based tools. It would also be opportune to consider a real-scale network, thereby obtaining a more accurate comparative analysis.

\section{Data Availability}

The data used to support the findings of this study are available from the corresponding author upon request.

\section{Conflicts of Interest}

The authors declare that they have no conflicts of interest.

\section{Acknowledgments}

This work was not supported by specific funding but was performed as part of the institutional activities of the authors in their university.

\section{References}

[1] R. Sacks, C. Eastman, G. Lee, and P. Teicholz, BIM Handbook: A Guide to Building Information Modeling for Owners, Designers, Engineers, Contractors, and Facility Managers, John Wiley \& Sons, Hoboken, NJ, USA, 3rd edition, 2018.

[2] A. Bradley, H. Li, R. Lark, and S. Dunn, "BIM for infrastructure: an overall review and constructor perspective," Automation in Construction, vol. 71, pp. 139-152, 2016.

[3] H. Chong, R. Lopez, J. Wang, X. Wang, and Z. Zhao, "Comparative analysis on the adoption and use of BIM in road infrastructure projects," Journal of Management in Engineering, vol. 32, pp. 1-13, 2016.

[4] G. Zhuo, "Application of BIM technology in airport topographical design," in Proceedings of the 2nd IEEE Advanced 
Information Management, Communicates, Electronic and Automation Control Conference (IMCEC), Xi'an, China, May 2018.

[5] F. Abbondati, S. A. Biancardo, S. Palazzo, F. S. Capaldo, and N. Viscione, "I-BIM for existing airport infrastructures," Transportation Research Procedia, vol. 45, pp. 596-603, 2020.

[6] S. A. Biancardo, N. Viscione, C. Oreto, R. Veropalumbo, and F. Abbondati, "BIM approach for modeling airports terminal expansion," Infrastructure, vol. 5, Article ID 5050041, 2020.

[7] S. A. Biancardo, M. Intignano, N. Viscione, S. Guerra De Olivera, and A. Tibaut, "Procedural modeling-based BIM approach for railway design," Journal of Advanced Transportation, vol. 2021, Article ID 8839362, 17 pages, 2021.

[8] F. Abbondati, S. A. Biancardo, G. Sicignano et al., "BIM parametric modelling of a railway underpass," Ingegneria Ferroviaria, vol. 6, pp. 443-459, 2020.

[9] F. Tschuchnigg and C. Lederhilger, "BIM in geotechnics - application to road and railway construction," in Proceedings of the International Conference on Information Technology in Geo-Engineering, Guimarães, Portugal, September 2019.

[10] M. Pasetto, A. Giordano, P. Borin, and G. Giacomello, "Integrated railway design using Infrastructure-Building Information Modeling. The case study of the port of Venice," Transportation Research Procedia, vol. 45, pp. 850-857, 2020.

[11] European Commission, https://ec.europa.eu/docsroom/ documents/34518/attachments/1/translations/en/renditions/ native European Construction Sector Observatory-Building Information Modelling in the EU Construction Sector, 2019.

[12] K. Shaaban and A. Nadeem, "Professionals' perception towards using building information modelling (BIM) in the highway and infrastructure projects," International Journal of Engineering Management and Economics, vol. 5, no. 3/4, pp. 273-289, 2015.

[13] A. H. Amer and O. Hasan, "Level of detail (LOD) specifications, standards and file-format challenges in infrastructure projects for BIM level three," WIT Transactions on the Built Environment, vol. 169, pp. 143-154, 2017.

[14] L. Chen, P. Shi, Q. Tang, W. Liu, and Q. Wu, "Development and application of a specification-compliant highway tunnel facility management system based on BIM," Tunnelling and Underground Space Technology, vol. 97, Article ID 103262, 2020.

[15] R. Bouška, "Evaluation of maturity of BIM tools across different software platforms," Procedia Engineering, vol. 164, pp. 481-486, 2016.

[16] C. Oreto, S. A. Biancardo, V. Nunzio, R. Veropalumbo, and R. Francesca, "Road pavement information modeling through maintenance scenario evaluation," Journal of Advanced Transportation, vol. 2021, Article ID 8823117, 14 pages, 2021.

[17] G. B. Ozturk, "Interoperability in building information modeling for AECO/FM industry," Automation in Construction, vol. 113, Article ID 103122, 2020.

[18] E. Cascetta, Transportation Systems Analysis: Models and Applications, Springer, New York, NY, USA, 2009.

[19] R. B. Potts and R. M. Oliver, Flows in Transportation Networks, Academic Press, New York, NY, USA, 1972.

[20] G. F. Newell, Traffic Flows in Transportation Networks, MIT Press, Cambridge, MA, USA, 1980.

[21] A. Radtke and R. Watson, "Railway simulation in the United Kingdom," Railway Technical Review, vol. 1, pp. 24-28, 2007.

[22] J. Barceló and J. Casas, "Dynamic network simulation with AIMSUN," in Simulation Approaches in Transportation Analysis. Operations Research/Computer Science Interfaces
Series, R. Kitamura and M. Kuwahara, Eds., Vol. 31, Springer, Boston, MA, USA, 2005.

[23] M. Behrisch, L. Bieker, J. Erdmann et al., "Evolution of SUMO's simulation model," in Traffic and Transportation Simulation-Looking Back and Looking Ahead: Celebrating 50 Years of Traffic Flow Theory, C. Antoniou, G. List, and R. L. Bertini, Eds., pp. 64-83, Transportation Research Board E-Circular, E-C195, Washington, DC, USA, 2014.

[24] D. Lin, X. Yang, and C. Gao, "VISSIM-based simulation analysis on road network of CBD in Beijing, China," Procedia - Social and Behavioral Sciences, vol. 96, pp. 461-472, 2013.

[25] B. D. Greenshields, "A study in highway capacity," Highway Research Board, vol. 14, pp. 448-477, 1935.

[26] Bureau of Public Roads, Traffic Assignment Manual, U. S. Department of Commerce, Urban Planning Division, Washington DC, USA, 1964.

[27] T. A. Domencich and D. McFadden, Urban Travel Demand: A Behavioural Analysis, American Elsevier, New York, NY, USA, 1975.

[28] M. Ben-Akiva and S. J. Lerman, Discrete Choice Analysis: Theory and Application to Travel Demand, The MIT Press, Cambridge, MA, USA, 1985.

[29] C. F. Daganzo and Y. Sheffi, "On stochastic models of traffic assignment," Transportation Science, vol. 11, no. 3, pp. 253-274, 1977.

[30] H. C. W. L. Williams, "On the formation of travel demand models and economic evaluation measures of user benefit," Environment \& Planning A: Economy and Space, vol. 9, no. 3, pp. 285-344, 1977.

[31] A. J. Daly and S. Zachary, "Improved multiple choice models," in Determinants of Travel Choice, D. A. Hensher and M. Q. Dalvi, Eds., Saxon House, Westmead, Australia, 1978.

[32] E. Cascetta, A. Nuzzolo, F. Russo, and A. Vitetta, "A modified logit route choice model overcoming path overlapping problems, specification and some calibration results for interurban networks," in Proceedings of the 13th International Symposium on Transportation and Traffic Theory, Lyon, France, July 1996.

[33] A. Papola, V. Marzano, and F. Tinessa, "Application of the combination of random utility models (CoRUM) to route choice," Transportation Research Part B: Methodological, vol. 111, pp. 304-326, 2018.

[34] C. F. Daganzo, Multinomial Probit: The Theory and its Application to Demand Forecasting, Academic Press, New York, NY, USA, 1979.

[35] J. L. Horowitz, J. M. Sparmann, and C. F. Daganzo, "An investigation of the accuracy of the clark approximation for the multinomial probit model," Transportation Science, vol. 16, no. 3, pp. 382-401, 1982.

[36] M. G. Langdon, "Improved algorithms for estimating choice probabilities in the multinomial probit model," Transportation Science, vol. 18, no. 3, pp. 267-299, 1984. 